\title{
Patients' Perception of Surgical Outcomes and Quality of Life after Retroperitoneoscopic and Open Pyeloplasty
}

\author{
Wael Y. Khoder ${ }^{a}$ Raphaela Waidelich ${ }^{a}$ Armin J. Becker ${ }^{a} \quad$ Alexander Karl $^{a}$ \\ Nicolas Haseke ${ }^{a}$ Ricarda M. Bauer ${ }^{a}$ Christian G. Stief ${ }^{a}$ Alexander Bachmann ${ }^{b}$ \\ Nicole Ebinger Mundorff ${ }^{b}$ \\ aDepartment of Urology, University Hospital Munich-Grosshadern, Ludwig Maximilian University Munich,

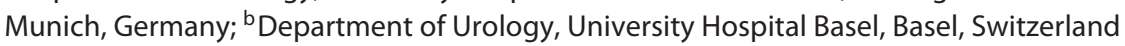

\section{Key Words}

Retroperitoneoscopic pyeloplasty · Pyeloplasty ·

Ureteropelvic junction obstruction - Open pyeloplasty

\begin{abstract}
Purpose: To report postoperative health-related quality of life (HRQoL) and patients' subjective evaluations of open pyeloplasty (OP) and retroperitoneoscopic pyeloplasty (RP) and influences on preoperative counselling. Methods: 107 patients (age 16-80 years, mean 31.5 ) with symptomatic primary ureteropelvic junction obstruction who underwent $\mathrm{OP}$ (32) or RP (75) were evaluated prospectively. HRQoL was evaluated using Short Form 36 (SF-36) questionnaires with 1 year follow-up. Operative outcomes were evaluated using a self-designed questionnaire regarding cosmetic outcomes, objective postoperative/current pain, convalescence and return to work. Results: The mean operative time was 174.4 vs. 161.4 min for RP versus OP, respectively, without intraoperative complications/conversions. There was an advantage for $\mathrm{RP}$ - except for two domains - without significance in any of the eight SF-36 domain scores. An advantage favouring RP in all aspects of the second questionnaire with significance in four aspects (cosmetic results, scar length, pain and con-
\end{abstract}

\section{KARGER}

E-Mail karger@karger.com

www.karger.com/uin valescence) was found. Five weeks postoperatively, 58.7\% (RP) vs. $25.8 \%(\mathrm{OP})$ were fully convalescent compared to $87.0 \%$ (RP) vs. $71.0 \%$ (OP) at 8 weeks. Similarly, 58.7 vs. $45.1 \%$ returned work 5 weeks postoperatively while 93.5 vs. $74.2 \%$ did so after 8 weeks, respectively. The small sample size, more questions on satisfaction/regret and mixed design are the main study limitations. Conclusion: RP provides the same functional results beside earlier convalescence, better HRQoL and patients' convenience with surgery, which favours its inclusion in preoperative counselling providing patients with realistic postoperative expectations.

Copyright $\odot 2013$ S. Karger AG, Basel

\section{Introduction}

Pyeloplasty is the standard surgery for ureteropelvic junction obstruction (UPJO) aiming to relieve symptoms and/or to preserve remaining renal function. Despite the high success rate (>95\%) of open pyeloplasty (OP) [1], minimally invasive techniques have been developed to

W.Y. Khoder and R. Waidelich shared senior authorship.
(C) 2013 S. Karger AG, Basel

0042-1138/13/0921-0074\$38.00/0
Dr. W.Y. Khoder, MD, PhD, FEBU

Urologische Klinik und Poliklinik, Klinikum Grosshadern Marchioninistrasse 15

DE-81377 München (Germany)

E-Mail wael.khoder@med.uni-muenchen.de 
lower its morbidity since the first successful OP reported by Kuster in 1891 [2].

Laparoscopic pyeloplasty (LP), initially reported by Kavoussi, has proved to be a safe and effective treatment for UPJO [3-5]. Retroperitoneoscopic pyeloplasty (RP) has evolved synchronously to LP with a success rate comparable to LP and OP [5]. The technique of LP/RP has been simplified over the years. Rising experience with this technique has made it safer, reproducible and standardized. The learning curve has become significantly shorter as more surgeons are familiar with these techniques [6$11]$.

Health-related quality of life (HRQoL) has become a major parameter in evaluating the outcome of a treatment and even constitutes a main criterion when choosing among different interventions with similar efficacy and morbidity [12]. The current study aims to compare surgical outcomes, HRQoL and patients' subjective evaluations of both standard approaches (OP and RP) to detect possible benefits of the procedures from the patients' point of view. This may be an addition to the preoperative counselling of these patients.

\section{Patients and Methods}

\section{Patients}

A total of 107 consecutive patients (64 male and 43 female, age 16-80 years, mean 31.5 years) with symptomatic primary UPJO, who underwent OP or RP, were evaluated for postoperative parameters and HRQoL. The surgical approach was OP (32 patients) and RP (75 patients), performed at the University Hospital of Basel (Switzerland) and the Ludwig Maximilian University Hospital in Munich (Germany). Both centres equally shared the patient collective. UPJO was treated in the left (72 cases) and right side (35 patients). OP was done according to patients' preference or before our experience with RP without any selection. Patients characteristics are presented in table 1.

Patients were assessed preoperatively by routine laboratory parameters, retrograde/intravenous pyelography and diuretic renography using ${ }^{99} \mathrm{Tc}$ mercaptoacetyltriglycine. All cases had one or more specific criteria of hugely dilated pelvis (42.0\% 2nd and $3 \mathrm{rd}$ grade, 45/107), crossing vessels at the ureteropelvic junction $(36.4 \%, 39 / 107)$ and/or compromised function of the affected kidney. Patients were operated retroperitoneally (open or endoscopic) under combined general and epidural anaesthesia.

\section{Operative Data}

The procedures were performed as described in the literature $[6,10]$. The patient was placed in a lateral position for both approaches. A flank lateral incision was used for the open approach. The skin and subcutaneous tissues were incised over tip of the 12th rib, extending along the superior border of the 12th $\mathrm{rib}(\approx 8 \mathrm{~cm})$. The latissimus dorsi, external oblique and internal oblique muscles were transected and the transversus abdominis was divided in the
Table 1. Demography of the included OP and RP patients

\begin{tabular}{lll}
\hline Characteristics & OP & RP \\
\hline Number of patients & 32 & 75 \\
Average age, years & $28.6(16-74)$ & $47.0(16-80)$ \\
Sex, M/F & $16 / 16$ & $48 / 27$ \\
Side, left/right & $10 / 22$ & $61 / 14$ \\
Median operative time, min & $161.4(40-365)$ & $174.4(70-360)$ \\
Average blood loss, ml & $160(90-360)$ & $180(50-200)$ \\
\hline
\end{tabular}

direction of its fibres with preservation of the intercostal neurovascular bundle. The transversalis fascia was divided with scissors near the tip of the 12th rib followed by blunt dissection of the peritoneum from the anterior abdominal wall. The Bookwalter ${ }^{\mathrm{TM}}$ retractor (Codman \& Shurtleff, Inc., Raynham, Mass., USA) was placed to retract the incision (11th rib and rib cage superiorly and 12 th rib and abdominal wall inferiorly). The retroperitoneal space was then developed through blunt and sharp dissection and the kidney with its surrounding soft tissues was reflected medially. The renal pelvis with the proximal ureter were then identified and dissected.

For the retroperitoneal approach, a 1-cm incision was made at the tip of last rib along the mid axillary line. The external, internal oblique and transversus abdominis muscle fibres were bluntly separated with a Kocher clamp. After the peritoneum had been digitally dissected medially from the abdominal wall and dorsally from the psoas muscle, a balloon made from the middle finger of a size 8 surgical glove and tied to the top of a $12-\mathrm{mm}$ trocar was placed in the retroperitoneal space and inflated with $500-700 \mathrm{ml}$ of saline to produce a working space. Inflation was maintained for a couple of minutes to allow haemostasis. In this manner, a working space to the upper retroperitoneum was obtained. The balloon was removed and the trocar was fixed in place using a 0 nylon suture to prevent carbon dioxide leakage. Carbon dioxide insufflation at a pressure of $13 \mathrm{~mm} \mathrm{Hg}$ was started to maintain the working space and diagnostic laparoscopy using a $30^{\circ}$ optic was done. The peritoneum was dissected from the anterior abdominal wall using the optic to insert another port $(12 \mathrm{~mm}$ for right-side or $5 \mathrm{~mm}$ to leftside pyeloplasty) in the anterior axillary line $2-3 \mathrm{~cm}$ above and medial to the anterior superior iliac spine. The second working port was made $1 \mathrm{~cm}$ from the middle point of the line between both ports. The whole procedure was done through these two ports.

The principle of Anderson-Hynes pyeloplasty was applied in all cases with trimming of redundant renal pelvis. Ureteropelvic re-anastomosis was performed by intracorporeal free hand continuous suturing using 4-0 polyglactin and laprotil clips [8]. A 7 -French ureter stent was placed retrogradely only at beginning of the series and removed 2-8 weeks postoperatively (median 4.2 for $\mathrm{OP}$ and 4.8 for RP). In patients with renal stones, ureter stents were left in place until complete fragmentation of the calculi $(n=12)$. Patients started oral intake on the first postoperative day and the urethral catheter was removed after $2-5$ days. The drainage tubes were removed after cessation of drainage/serous discharge. Operative time (from introduction of the first port till closure of last port site), intraoperative and postoperative complications were recorded. 
Follow-Up

HRQoL was evaluated using the Short Form 36 (SF-36) questionnaire with follow-up of 1 year. It has been specially validated for the German-speaking population (IQOLA-SF-36 German version 1992) and has been used to evaluate possible body limitations through physical health problems, body pains, general health, vitality, social functioning, emotional problems and mental health. The Mental Component Summary Score and the Physical Component Summary Score are calculated according to a given SF-36 syn$\operatorname{tax}[13]$.

Furthermore, operation-related outcomes were evaluated using a self-designed questionnaire. Patients were asked a set of 20 questions $(\mathrm{Q})$ regarding cosmetic outcomes $(8 \mathrm{Q})$, postoperative symptoms/complications (3Q), objective postoperative pain [early (3Q) and current situation (1Q)], convalescence (1Q), time to return to work (1Q), acceptance of operation (1Q), fluid intake (1Q) and follow-up intervals (1Q).

\section{Results}

Presenting symptoms were renal flank pain $(49.5 \%$, 53/107), colic without evidence of nephrolithiasis (9.3\%, $10 / 107)$, recurrent pyelonephritis $(15.9 \%, 17 / 107)$ and nephrolithiasis $(12.0 \%, 13 / 107)$ while $13.0 \%(14 / 107)$ were asymptomatic.

The diagnosis of anatomical/functional UPJO was documented preoperatively through abdominal ultrasound (107 patients, $100 \%)$, abdominal CT $(32,29.9 \%)$, MRI (5, 4.7\%), intravenous pyelography $(21,19.6 \%)$ and retrograde pyelography $(87,81.3 \%)$. Renal scintigraphy revealed ipsilateral renal function of 19-24 in 7.5\%, 25-29 in $12.1 \%, 29-39$ in $28.0 \%, 40-49$ in $35.5 \%$ and $>50$ in $16.8 \%$ of patients, respectively. Renal glomerular filtration rates as well as total function were within the ageadapted normal range. Preoperative nephrostomy tube insertion was necessary in 2 emergency patients while a double- ${ }^{\circledR}$ catheter was inserted electively.

Operative time was 70-360 min (mean 174.4) and 40365 min (mean 161.4) with a median blood loss of 180 and $160 \mathrm{ml}$ for RP and OP, respectively. Crossing vessels $(36.4 \%, 39 / 107)$, obstructing adhesions/ureteric scars $(18.7 \%, 20 / 107)$ and primary UPJO $(44.9 \%, 48 / 107)$ were found. Postoperative complications were ureter stent dislocation $(\mathrm{n}=1)$, temporary nephrostomy for hydronephrosis $(\mathrm{n}=1)$, which was successfully treated conservatively without need of re-operation, and epididymitis $(\mathrm{n}=1)$.

77 patients (31 OP and $46 \mathrm{RP}$ ) returned their SF-36 questionnaire. A standardized scale allowed the patients' answers to be assigned to an individual domain score. This was translated to a scale of $0-100(0=$ very poor,
$100=$ excellent). Clinical relevance starts from a difference of at least 20 points. Results are shown in figure 1 and table 2 .

An advantage in favour of $\mathrm{RP}$ was seen in six of eight domain scores (physical functioning, general health problems, vitality, social functioning, emotional role function, mental health). In two domains (limitations caused by physical health and bodily pains), the OP results were slightly better than those of RP. However, neither group showed any significant difference in any of SF-36 domain scores.

Table 3 demonstrates all results of the second questionnaire. A large proportion of this questionnaire evaluated the cosmetic outcomes of surgery. $25.0 \%$ of patients claimed to be 'concerned' about cosmetic results preoperatively. $23.5 \%$ reported that this outcome played a 'minor role', and $51.5 \%$ were 'not worried' about the cosmetic result (19.6\% of them reported that the importance of this issue increased over time postoperatively).

Patients reported different grades of satisfaction regarding their cosmetic results: 26.0 vs. $22.5 \%$ 'excellent', 39.1 vs. $35.5 \%$ 'very good', 19.6 vs. $19.4 \%$ 'good', 17.4 vs. 9.7\% 'slightly bad' and 2.2 vs. $9.7 \%$ 'bad' for RP versus OP, respectively. Furthermore, 50.0 vs. $48.4 \%, 39.1$ vs. $22.6 \%$ and 3.2 vs. $10.9 \%$ of RP versus OP patients reported that they were 'not disturbed', 'slightly disturbed' and 'disturbed' with their scars, respectively, while 0 vs. $25.8 \%$ reported to be 'much disturbed' for RP versus OP, respectively.

Postoperative scar length was reported to be between 7 and $30 \mathrm{~cm}$ (average $13.9 \mathrm{~cm}$ ) for OP. This was judged as 'small' (54.8\%), 'moderate' (16.1\%), 'large' (19.4\%) and 'very large' $(9.7 \%)$ by patients. The reported sum scar lengths of RP patients were between 2.5 and $7 \mathrm{~cm}$ (average $3.7 \mathrm{~cm}$ ), which were judged as 'small' $(60.9 \%)$, 'moderate' (39.1\%), 'large' (0\%) and 'very large' (0\%). Patients evaluated the location of their scar(s) as 'not disturbing' (67.4 vs. $28.4 \%$ ), 'slightly disturbing' (26.0 vs. $25.8 \%$ ) and 'disturbing' (4.3 vs. $22.6 \%$ ) for RP versus OP, respectively.

A second set of questions aimed to evaluate early/current ( 1 year) postoperative pain. 45.7 vs. $16.1 \%$ of patients claimed that the operation was 'not painful', 32.6 vs. $45.1 \%$ 'slightly painful', 13.0 vs. $16.1 \%$ 'painful', while 8.7 vs. $22.7 \%$ judged it as 'very painful' for RP versus OP, respectively. Surgical scars were reported by 69.6 vs. $67.7 \%$ as 'painless', 21.7 vs. $16.1 \%$ 'slightly painful', 8.7 vs. $16.1 \%$ 'painful' and 0 vs. $0 \%$ 'severely painful' for RP and OP at evaluation, respectively. Furthermore, patients were 


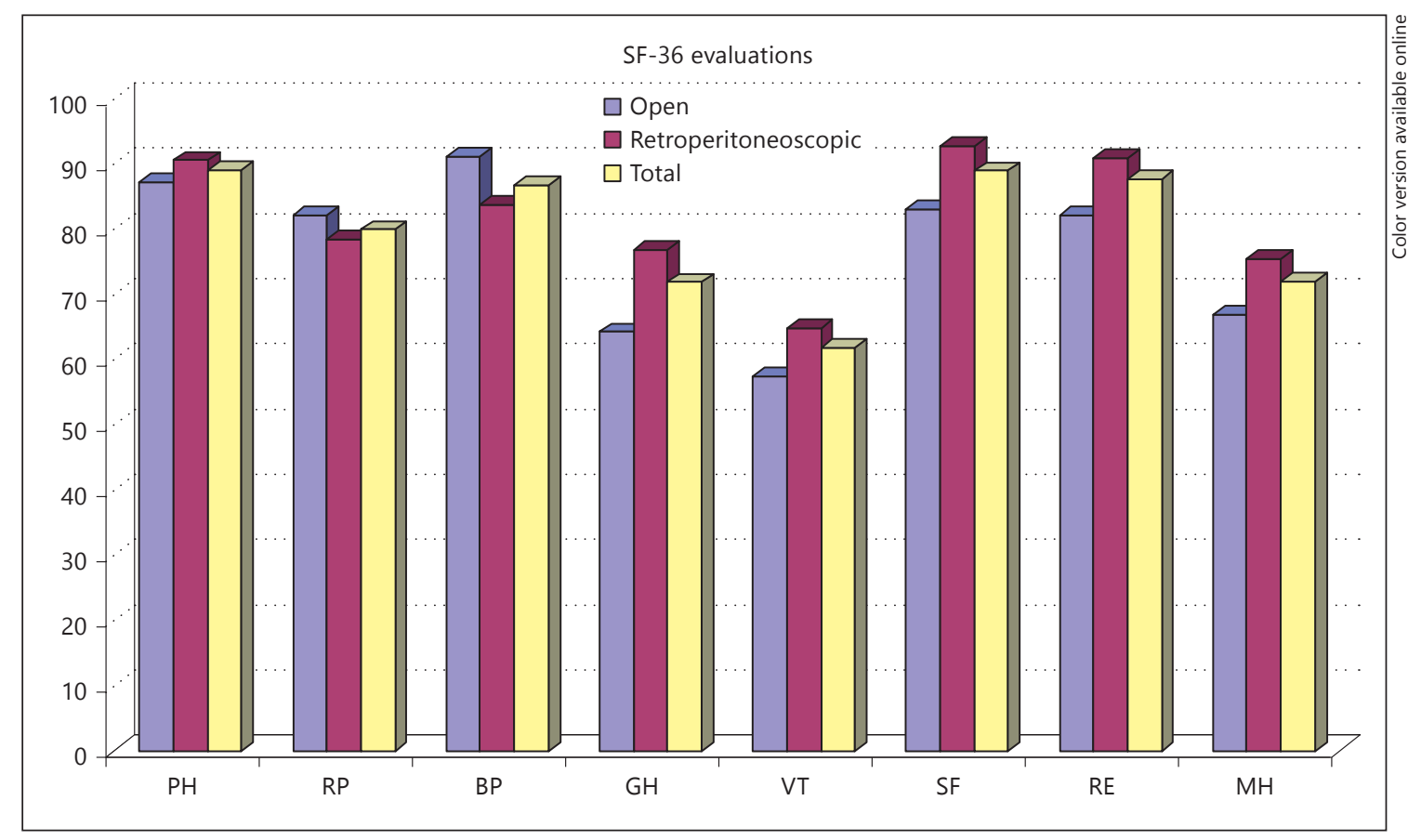

Fig. 1. Results of the eight domain scores of the SF-36 in patients after OP and RP versus the total collective. $\mathrm{PH}=$ Physical functionality; RP = role limitations caused by physical health problems; $\mathrm{BP}=$ bodily pain; $\mathrm{GH}=$ general health; $\mathrm{VT}=$ vitality; $\mathrm{SF}=$ social functioning; $\mathrm{RE}=$ role limitation caused by emotional problems; $\mathrm{MH}=$ mental health. Furthermore the Mental Component Summary Scores were calculated. Scores per dimension range from 0 to 100; higher scores indicate better health status.

Table 2. Mean and standard deviation (SD) of the results of the SF-36 for total operations, OP and RP

\begin{tabular}{lllllll}
\hline & $\begin{array}{l}\text { Mean } \\
\text { all }\end{array}$ & $\begin{array}{l}\text { SD } \\
\text { all }\end{array}$ & $\begin{array}{l}\text { Mean } \\
\text { OP }\end{array}$ & $\begin{array}{l}\text { SD } \\
\text { OP }\end{array}$ & $\begin{array}{l}\text { Mean } \\
\text { RP }\end{array}$ & $\begin{array}{l}\text { SD } \\
\text { RP }\end{array}$ \\
\hline Physical functionality & 89.1 & 17.5 & 87.1 & 19.8 & 90.5 & 15.7 \\
Role limitations caused by physical health problems & 79.9 & 37.2 & 82.1 & 35.9 & 78.4 & 37.9 \\
Bodily pain & 86.7 & 16.6 & 91.0 & 12.9 & 83.7 & 18.2 \\
General health & 71.8 & 20.5 & 64.2 & 15.5 & 76.8 & 21.8 \\
Vitality & 61.8 & 20.3 & 57.3 & 24.5 & 64.8 & 16.1 \\
Social functioning & 88.9 & 16.9 & 83.0 & 19.8 & 92.6 & 13.4 \\
Role limitations caused by emotional problems & 87.6 & 25.3 & 82.1 & 31.0 & 90.9 & 20.6 \\
Mental health & 71.9 & 16.6 & 66.7 & 17.6 & 75.4 & 14.9 \\
\hline
\end{tabular}

The Mental Component Summary Scores were calculated. Scores per dimension range from 0 to 100; higher scores indicate better health status.

asked to evaluate their pain level on a scale from 0 ('absolutely painless') to 10 ('severe pain') (fig. 2).

Another question was whether patients felt increasingly ill because of the operation. 4.3 vs. $12.9 \%$ answered 'yes', 89.1 vs. $83.9 \%$ 'no' and 6.5 vs. $3.2 \%$ with 'I do not know' of RP versus OP patients.

Patients' Perception of Outcomes and Quality of Life after Pyeloplasty
The last two questions aimed to find out how soon patients were fully convalescent postoperatively and when they were back to work. Five weeks postoperatively, $58.7 \%(\mathrm{RP})$ vs. $25.8 \%(\mathrm{OP})$ of patients were fully convalescent compared to $87.0 \%(\mathrm{RP})$ vs. $71.0 \%(\mathrm{OP})$ at 8 weeks. Similarly, 58.7 vs. $45.1 \%$ returned to work at 
Table 3. Results of the self-made life quality questionnaire in patients post RP versus OP

Parameter/questions RP versus OP

Cosmetic results

How satisfied are you with the cosmetic results of surgery?

Are you disturbed by your scar(s)?

Did the position of scar(s) disturb you?

Did you think that your scar(s) are long?

Were you concerned about the cosmetic results of surgery?

Did cosmetic results become more important to you with time postoperatively?

\begin{tabular}{|c|c|c|c|c|}
\hline Excellent & Very good & Good & Slightly bad & $\mathrm{Bad}$ \\
\hline 26.0 vs. $22.5 \%$ & 39.1 vs. $35.5 \%$ & 19.6 vs. $19.4 \%$ & 17.4 vs. $9.7 \%$ & 2.2 vs. $9.7 \%$ \\
\hline Not disturbed & Slightly disturbed & Disturbed & Much disturbed & \\
\hline 50.0 vs. $48.4 \%$ & 39.1 vs. $22.6 \%$ & 3.2 vs. $10.9 \%$ & 0 vs. $25.8 \%$ & \\
\hline 67.4 vs. $28.4 \%$ & 26.0 vs. $25.8 \%$ & 4.3 vs. $22.6 \%$ & 2.3 vs. $23.2 \%$ & \\
\hline Small & Moderate & Large & Very large & \\
\hline 60.9 vs. $54.8 \%$ & 39.1 vs. $16.1 \%$ & 0 vs. $19.4 \%$ & 0 vs. $9.7 \%$ & \\
\hline Concerned & Minor role & Not worried & & \\
\hline $25.0 \%$ & $23.5 \%$ & $51.5 \%$ & & \\
\hline No & Little & More than little & Yes & \\
\hline $\begin{array}{c}81.2 \text { vs. } 78.6 \% \\
3.7 \text { vs. } 13.9\end{array}$ & 18.2 vs. $14.3 \%$ & 6.0 vs. $7.2 \%$ & 0 vs. $0 \%$ & \\
\hline Painless & Slightly painful & Painful & Very painful & \\
\hline 45.7 vs. $16.1 \%$ & 32.6 vs. $45.1 \%$ & 13.0 vs. $16.1 \%$ & 8.7 vs. $22.7 \%$ & \\
\hline 37.3 vs. $14.2 \%$ & 50.0 vs. $28.6 \%$ & 4.6 vs. $14.3 \%$ & 9.0 vs. $42.9 \%$ & \\
\hline $\begin{array}{l}69.6 \text { vs. } 67.7 \% \\
\text { figure } 2 b\end{array}$ & 21.7 vs. $16.1 \%$ & 8.7 vs. $16.1 \%$ & 0 vs. $0 \%$ & \\
\hline
\end{tabular}

How long are your scars? (average in $\mathrm{cm}$ )

Pains

Did you have pains/complications early postoperatively?

Was the operation a painful event for you?

Do you have scar pain now?

figure $2 \mathrm{~b}$

Please evaluate your pain in a scale from 1 to 10

Postoperative symptoms/complications

Did you still have any symptoms similar to these at presentation before surgery?

No Yes

100 vs. $100 \% \quad 0$ vs. $0 \%$

UTI Stones No

Did you have urinary tract infections or recurrent kidney stones in the same side?

3.7 vs. $13.9 \% \quad 0$ vs. $0 \%$

96.3 vs. $86.1 \%$

Convalescence

When were you completely convalescent?

At 5 weeks At 8 weeks

58.7 vs. $25.8 \% \quad 87$ vs. $71 \%$

Return to work

When did you return to work?

At 5 weeks At 8 weeks

58.7 vs. $45.1 \% \quad 93.5$ vs. $74.2 \%$

Recurrence

Have you had a re-pyeloplasty operation?

No Yes

100 vs. $100 \% \quad 0$ vs. $0 \%$

Acceptance of the procedure

Did you have a feeling of being ill because of the operation?

No Yes I do not know

89.1 vs. $83.9 \% \quad 4.3$ vs. $12.9 \% \quad 6.5$ vs. $3.2 \%$

Follow-up

Are you satisfied with the follow-up examinations and intervals?

How much did you drink per day?

$\begin{array}{ll}\text { Yes } & \text { No } \\ 100 \text { vs. } 100 \% & 0 \text { vs. } 0 \% \\ \leq 2 \text { l/day }(45.1 \%) & >2 \text { l/day }(54.9 \%)\end{array}$




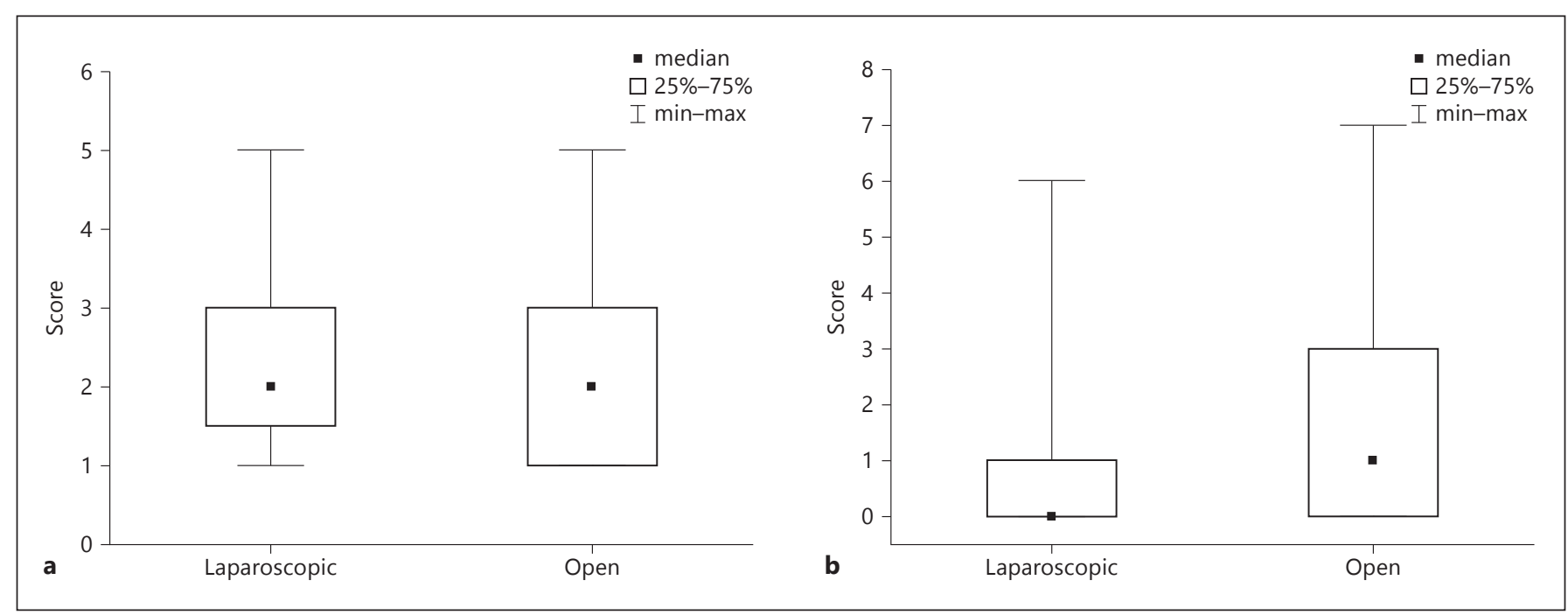

Fig. 2. Evaluations of the patients' answers to some questions of the second questionnaire. a 'How much are you satisfied with the cosmetic results regarding your scars?' b 'Please describe your current pains, if present, using the following scale between 0 (no pain) and 10 (very strong pain)'.

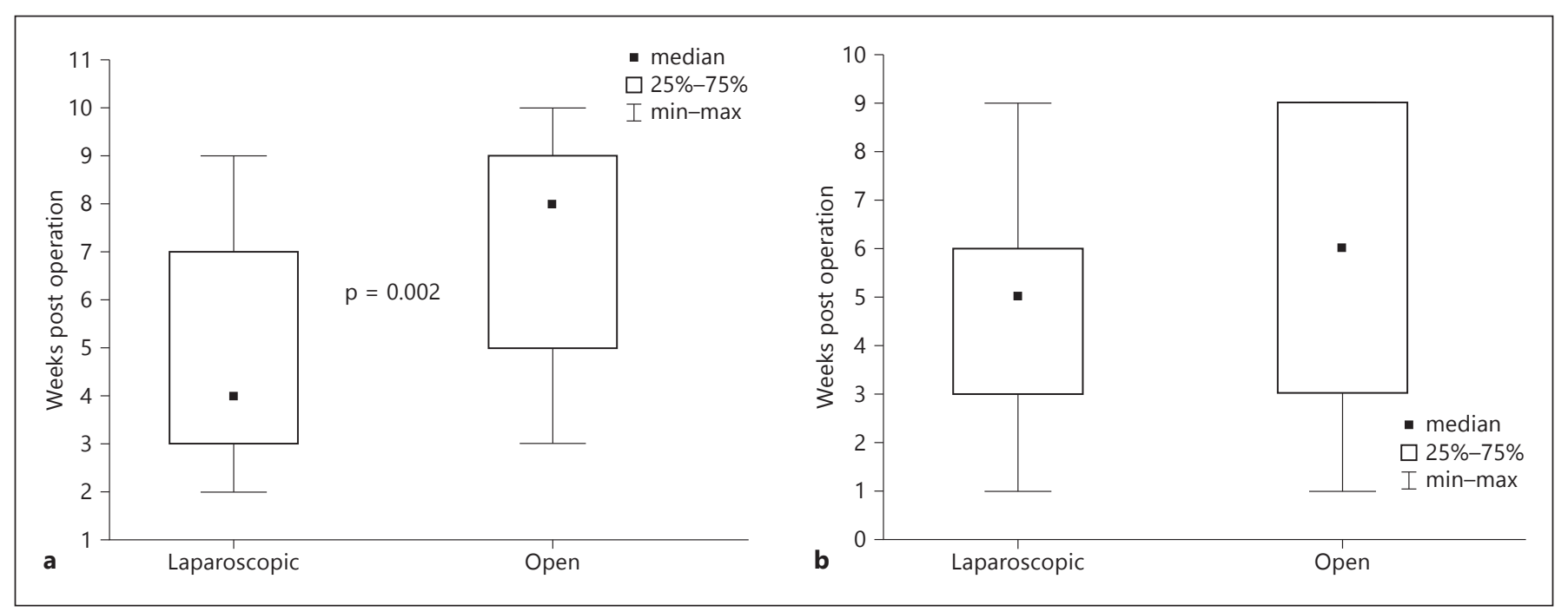

Fig. 3. Evaluations of the patients' answers to questions of the second questionnaire regarding convalescence and return to work. a Convalescence period: 'How long did it take after the operation to be again completely (100\%) physically normal?' b 'When did you returned back to your work after the operation?'.

5 weeks while 93.5 vs. $74.2 \%$ returned after 8 weeks, respectively (fig. 3).

Univariate analysis revealed an advantage to RP over $\mathrm{OP}$ with a significant difference in five questions related to the cosmetic results, scar length, postoperative pains and postoperative convalescence results.

Patients' Perception of Outcomes and Quality of Life after Pyeloplasty

\section{Discussion}

A major aim of any reconstructive surgery is to improve patients' quality of life. Clinicians often estimate an intervention to be successful if it solves the primary obstruction. Surgeons are able to characterize the result of 
pyeloplasty with objective tests such as postoperative radiography/renography. However, measuring HRQoL can add another dimension to the surgical outcomes, providing patients with the best quality of health care.

The present study shows for the first time the impact of two standard surgical procedures (OP and RP) in terms of HRQoL as well as subjective patient evaluation over an intermediate follow-up time. Both participating centres consider RP as a standard UPJO management. Generally, the literature data support that RP combines the highly successful OP results and the quick postoperative recovery after laparoscopy [14-17].

Laparoscopic procedures have been associated with lower plasma levels of cytokines like IL-1, IL-6, IL-10 and CRP compared with conventional resections. The extent of this reaction to operative trauma correlates more with the approach than with the extent of the procedure. Renal surgeries trigger a systemic acute phase reaction which can be limited by the laparoscopic access [18]. These mild systemic reactions may be responsible for the rapid postoperative convalescence $[18,19]$.

The current surgical and functional outcomes are comparable with previous studies [5-10, 14-17]. Furthermore, RP proved to be superior for intraoperative blood loss and early postoperative complications, although operative time was longer. Functional results are comparable between the two groups. Importantly, patients report high HRQoL independent from the technique used, which is a logical reflection of surgical healthy patients. Only $4.3 \%$ (RP) vs. $12.9 \%$ (OP) of patients had a feeling of being 'slightly ill' because of the operation.

There is no known standard questionnaire for the evaluation of such cases. The mentioned self-made broadspectrum questionnaire was designed to evaluate all operation-related aspects in these patients. Generally, RP patients reported better results in all evaluated aspects (cosmetic, postoperative pain (early/current), convalescence, return to work) than OP patients. RP patients expressed their satisfaction with cosmetic results as a clear advantage over OP ( 28.0 vs. $14.0 \%$, respectively). On the other hand, 19.4 and $9.7 \%$ of OP patients reported their frustration regarding scar length (being long and very long, respectively) and 22.6 and $23.2 \%$ regarding scar location (being disturbed and much disturbed, respectively).

Another advantage for RP was postoperative and current (1 year) pains. Early pain was reported by $8.7 \%(\mathrm{RP})$ vs. $22.7 \%(\mathrm{OP})$ as 'strong pain', while current scar pain was reported by $8.7 \%(\mathrm{RP})$ vs. $16.1 \%(\mathrm{OP})$ patients.
A notable benefit is the significant difference in the convalescence period. At 5 weeks, $58.7 \%$ (RP) vs. $25.8 \%$ (OP) felt 'fully convalescent' again. This was better at 8 weeks, reaching $87.0 \%$ (RP) vs. $71.0 \%$ (OP). Accordingly, return to work was reported in $58.7 \%(\mathrm{RP})$ and $45.1 \%$ (OP) after 5 weeks postoperatively, while in $93.5 \%$ (RP) and $74.2 \%(\mathrm{OP})$ it was after 8 weeks.

There was no significant difference in any of the eight SF-8 domain scores, although RP revealed superior results in six domains compared to OP (statistically insignificant). Possible explanations are the timing of the HRQoL assessment, small sample size or lack of the calculations' power provided. Following conventional upper urinary tract surgery, complete recovery usually takes several weeks to months. With the advent of laparoscopy, these times have been significantly shortened [20]. Accordingly, the SF-8 questionnaire should have been administered at an earlier time point or at different followup time points to observe the gradual improvement in physical health.

Further, comparing the outcomes between open and laparoscopic nephrectomy (simple, radical and donor) using the postoperative recovery scale (modified SF-36 for evaluation of post-nephrectomy patients) revealed that postoperative HRQoL in both groups did not reach baseline values until at least 1 year. Laparoscopic patients did better than open surgery patients at each time point assessed [21]. Current 1-year results confirm that HRQoL does not seem to be negatively affected in both procedures.

A previous study evaluating HRQoL after laparoscopic urinary tract surgery for malignant and benign conditions showed no significant difference between pre- and postoperative SF-8 evaluations [22]. However, the patient groups were heterogeneous, which limits interpretation for specific surgical approaches. In the current series, patients were asked more detailed questions about surgery in order to evaluate all aspects from the patients' point of view.

The current data support that RP is not only safe, but also leads to good postoperative HRQoL. These observations confirm the minimal invasiveness of RP over OP from the patients' point of view and encourage the addition of this procedure, as a valuable therapy, to the preoperative counselling of patients.

Patient self-evaluation has been previously reported for other postoperative functions to have reliability and discriminating power $[23,24]$. Further financial analyses will be necessary to quantify the impact of RP on workplace productivity and the indirect costs borne by patients
Khoder/Waidelich/Becker/Karl/Haseke/ Bauer/Stief/Bachmann/Ebinger Mundorff 
and employers. However, the current information is of outmost value to case managers, patients and clinicians aligned on reducing morbidity and hastening early return to normal work. There is for sure a financial impact of the clearly shown differences in rehabilitation time on the reduction of costs and the gain in productivity (for both health system and patients work) based on the current results for RP.

The current study has limitations, as for instance the difference in the idiosyncrasies of the two national health systems. The mixed prospective and retrospective design may have allowed for a certain self-selection bias, however, patients were enrolled in a consecutive series. Furthermore, the nature of the study neither allows for the assessment of HRQoL at specified postoperative time points nor for evaluations of change from baseline HRQoL. Our intention was to demonstrate the patients' subjective evaluations of these standard techniques. In addition to small sample size, more questions on satisfaction and regret could have provided more aspects regarding outcomes. This issue needs validation in future prospective studies.

Despite these limitations, we think that our findings are robust. Currently, a prospective randomized trial comparing minimally invasive versus open pyeloplasty is unethical and not feasible. The studied groups were strictly comparable in all aspects, omitting biases that could compromise the broadness of the conclusions reached. This was the first time a study addressed outcome measures from the patients' point of view.
However, pain and convalescence are objective feelings which can be differently interpreted by different patients. Treatment satisfaction is mainly derived from perceived differences between expectations and experience [25]. Expectations of one's future health state have been shown to influence patients' reported HRQoL [26]. Thus patients who choose the 'less invasive' RP may have higher expectations for their postoperative HRQoL compared to patients choosing 'traditional' surgery. Therefore, even when they achieve similar functional outcomes with better evaluation score results compared to OP patients, they may still experience a higher level of dissatisfaction. This could explain the current results showing a statistical significance in only five questions between the two groups. Based on these results, preoperative patient counselling should provide them with realistic expectations for the postoperative results to avoid dissatisfaction.

\section{Conclusion}

The follow-up results and patient evaluations revealed complete patient satisfaction with $\mathrm{RP}$ with a better HRQoL versus OP. A loin wound is arguably not necessary for either a successful outcome or patients' convenience after surgery. Preoperative counselling should include RP and provide patients with realistic expectations for the time after surgery.

\section{References}

- 1 Brooks JD, Kavoussi LR, Preminger GM, Schuessler WW, Moore RG: Comparison of open and endourologic approaches to the obstructed ureteropelvic junction. Urology 1995;46:791-795.

2 Murphy LJT: The kidney; in Murphy LJT (ed): The History of Urology. Springfield, Charles C. Thomas, 1972, pp 201-232.

-3 Kavoussi LR, Peters CA: Laparoscopic pyeloplasty. J Urol 1993;150:1891-1894.

-4 Singh O, Gupta SS, Hastir A, Arvind NK: Laparoscopic dismembered pyeloplasty for ureteropelvic junction obstruction: experience with 142 cases in a high-volume center. J Endourol 2010;24:1431-1434.

-5 Chuanyu S, Guowei X, Ke X, Qiang D, Yuanfang Z: Retroperitoneal laparoscopic dismembered Anderson-Hynes pyeloplasty in treatment of ureteropelvic junction obstruction (report of 150 cases). Urology 2009;74: 1036-1040.

Patients' Perception of Outcomes and Quality of Life after Pyeloplasty
Rassweiler JJ, Seemann O, Frede T, Henkel TO, Alken P: Retroperitoneoscopy: experience with 200 cases. J Urol 1998;160:1265-1269.

$\checkmark 7$ Ben Slama MR, Salomon L, Hoznek A, Cicco A, Saint F, Alame W, Antiphon P, Chopin DK, Abbou CC: Extraperitoneal laparoscopic repair of ureteropelvic junction obstruction: initial experience in 15 cases. Urology 2000; 56:45-48.

8 Bachmann A, Ruszat R, Forster T, Eberli D, Zimmermann M, Müller A, Gasser TC, Sulser T, Wyler S: Retroperitoneoscopic pyeloplasty for ureteropelvic junction obstruction (UPJO): solving the technical difficulties. Eur Urol 2006;49:264-272.

-9 Bonnard A, Fouquet V, Carricaburu E, Aigrain Y, El-Ghoneimi A: Retroperitoneal laparoscopic versus open pyeloplasty in children. J Urol 2005;173:1710-1713.

10 Wyler SF, Bachmann A, Casella R, Gasser T, Sulser T: Retroperitoneoscopic pyeloplasty for ureteropelvic junction obstruction. J Endourol 2004; 18:948-951.

11 Tan BJ, Rastinehad AR, Marcovich R, Smith $A D$, Lee BR: Trends in ureteropelvic junction obstruction management among urologists in the United States. Urology 2005;65:260-264.

12 Cella D, Yount S, Du H, Dhanda R, Gondek K, Langefeld K, George J, Bro WP, Kelly C, Bukowski R: Development and validation of the Functional Assessment of Cancer Therapy-Kidney Symptom Index (FKSI). J Support Oncol 2006;4:191-199.

$\checkmark 13$ Gratzke C, Seitz M, Bayrle F, Schlenker B, Bastian PJ, Haseke N, Bader M, Tilki D, Roosen A, Karl A, Reich O, Khoder WY, Wyler S, Stief CG, Staehler M, Bachmann A: Quality of life and perioperative outcomes after retroperitoneoscopic radical nephrectomy (RN), open $\mathrm{RN}$ and nephron-sparing surgery in patients with renal cell carcinoma. BJU Int 2009; 104:470-475. 
14 Eden CG: Minimally invasive treatment of ureteropelvic junction: a critical analysis of results. Eur Urol 2007;52:983-989.

15 Davenport K, Minervin A, Timoney AG, Keeley FX Jr: Our experience with retroperitoneal and transperitoneal laparoscopic pyeloplasty for pelvi-ureteric junction obstruction. Eur Urol 2005;48:973-977.

16 Imkamp F, Hermann TR, Rassweiler J, Sulser T, Stolzenburg JU, Rabenalt R, Jonas U, Burchardt M: Laparoscopy in German urology: changing acceptance among urologists. Eur Urol 2009;56:1074-1080.

-17 Shao P, Qin C, Ju X, Meng X, Li J, Lv Q, Zhang $\mathrm{W}, \mathrm{Xu} \mathrm{Z}$, Yin C: Comparison of two different suture methods in laparoscopic dismembered pyeloplasty. Urol Int 2011;87:304-308.

-18 Fornara P, Doehn C, Seyfarth M, Jocham D: Why is urological laparoscopy minimally invasive? Eur Urol 2000;37:241-250.
9 Schwenk W, Neudecker J, Mall J, Böhm B, Müller JM: Prospective randomized blinded trial of pulmonary function, pain, and cosmetic results after laparoscopic vs microlaparoscopic cholecystectomy. Surg Endosc 2000; 14:345-348.

20 Rhaghuram S, Godbole HC, Dasgupta P: Laparoscopic nephrectomy: the new gold standard? Int J Clin Pract 2005;59:128-129.

21 Pace KT, Dyer SJ, Stewart RJ, Honey RJ, Poulin EC, Schlachta CM, Mamazza J: Health-related quality of life after laparoscopic and open nephrectomy. Surg Endosc 2003;17: 143-152.

22 Sahai A, Tang S, Challacombe B, Murphy D, Dasgupta P: Laparoscopic upper urinary tract surgery for benign and malignant conditions. Does aetiology have an effect on health-related quality of life? Int J Clin Pract 2007;61: 2026-2029.
3 Casella R, Deckart A, Bachmann A, Sulser T, Gasser TC, Lehmann K: The patient's selfevaluation better predicts the degree of erectile dysfunction than the response to intracavernous alprostadil testing. Urol Int 2004;72: 216-220.

24 Froehner M, Koch R, Leike S, Novotny V, Twelker L, Wirth MP: Urinary tract-related quality of life after radical prostatectomy: open retropubic versus robot-assisted laparoscopic approach. Urol Int 2013;90:36-40.

25 Pascoe GC: Patient satisfaction in primary health care: a literature review and analysis. Eval Program Plann 1983;6:185-210.

26 Llewellyn-Thomas HA, Thiel EC, McGreal MJ: Cancer patients' evaluations of their current health states: the influences of expectations, comparisons, actual health status, and mood. Med Decis Making 1992;12: 115-122. 